\title{
First-passage statistics for aging diffusion in systems with annealed and quenched disorder
}

\author{
Henning Krüsemann, ${ }^{1}$ Aljaž Godec,,${ }^{1,2}$ and Ralf Metzler ${ }^{1,3, *}$ \\ ${ }^{1}$ Institute of Physics \& Astronomy, University of Potsdam, 14776 Potsdam-Golm, Germany \\ ${ }^{2}$ National Institute of Chemistry, 1000 Ljubljana, Slovenia \\ ${ }^{3}$ Department of Physics, Tampere University of Technology, FI-33101 Tampere, Finland
}

(Received 23 January 2014; published 28 April 2014)

\begin{abstract}
Aging, the dependence of the dynamics of a physical process on the time $t_{a}$ since its original preparation, is observed in systems ranging from the motion of charge carriers in amorphous semiconductors over the blinking dynamics of quantum dots to the tracer dispersion in living biological cells. Here we study the effects of aging on one of the most fundamental properties of a stochastic process, the first-passage dynamics. We find that for an aging continuous time random walk process, the scaling exponent of the density of first-passage times changes twice as the aging progresses and reveals an intermediate scaling regime. The first-passage dynamics depends on $t_{a}$ differently for intermediate and strong aging. Similar crossovers are obtained for the first-passage dynamics for a confined and driven particle. Comparison to the motion of an aged particle in the quenched trap model with a bias shows excellent agreement with our analytical findings. Our results demonstrate how first-passage measurements can be used to unravel the age $t_{a}$ of a physical system.
\end{abstract}

DOI: 10.1103/PhysRevE.89.040101

PACS number(s): 05.40.-a, 72.20.Jv, 72.70.+m, 89.75.Da

Introduction. In their groundbreaking 1975 paper, Scher and Montroll introduced the scale-free distribution $\psi(\tau) \simeq$ $\tau^{-1-\alpha}(0<\alpha<1)$ of trapping times $\tau$ for moving charge carriers in amorphous semiconductors to explain the measured power-law form of the electrical current [1]. This then radical assumption has since been observed in other semiconductor materials [2] and found to be the defining property of the dynamics in numerous other systems [3], including the tracer dispersion in groundwater aquifers [4], the motion of endogenous protein channels and granules in biological cells $[5,6]$ and of submicron tracers in structured environments [7], or the blinking dynamics of quantum dots [8] and subrecoil laser cooling [9].

The lack of a characteristic time scale $\langle\tau\rangle$ in these systems effects the disparity between ensemble and time averages of physical observables [10,11] and an explicit dependence of observables on the time span between the initial preparation of the system and start of the measurement at $t_{a}$, the so-called aging [12-15]. Aging phenomena can be rationalized by particle dynamics in quenched energy landscapes [16] or logistic maps [17], and were experimentally observed in biological systems [5] as well as amorphous semiconductors [18]. It is typical in these experiments that the aging time $t_{a}$ is comparable to or longer than the actual observation time $[5,18]$.

Here we present analytical and numerical evidence for the distinct effects of aging on the first-passage properties of aging systems, i.e., the statistics of the times when the process first crosses a preset value. In the above examples, this is the arrival of charge carriers at the counterelectrode, giving rise to the decay of the electrical current, the breakthrough of chemical tracers at some probe location in an aquifer, the arrival of a protein channel at a specific receptor in a cell membrane, or for a quantum dot to reach its $n$th on-state. Our results for the first-passage time density (FPTD) $\wp(t)$ in systems with

*rmetzler@uni-potsdam.de a diverging time scale $\langle\tau\rangle$ exhibit a clear dependence on the aging time $t_{a}$ in the cases of intermediate and strong aging, while $\wp(t)$ is independent of $t_{a}$ for short aging times. More importantly, progressing aging changes the scaling exponent of $\wp(t)$ and reveals an interesting intermediate scaling regime. For biased diffusion, we show that our results agree well with simulations in a quenched energy landscape. Finally, we address the question of how first-passage measurements can be used to unravel information on the age $t_{a}$ of the observed system.

In the absence of aging $\left(t_{a}=0\right)$, i.e., when the measurement commences simultaneously with the initiation of the system at $t=0$, the Scher-Montroll continuous time random walk (CTRW) process with trapping time density $\psi(\tau) \simeq \tau^{-1-\alpha}$ and $0<\alpha<1$ causes free anomalous diffusion of the subdiffusive form $\left\langle x^{2}(t)\right\rangle \simeq K_{\alpha} t^{\alpha}$, with the anomalous diffusion coefficient $K_{\alpha}[1,3]$. In contrast, when the system has aged for the period $t_{a}$, the mean squared displacement crosses over from $\left\langle x^{2}(t)\right\rangle \simeq$ $K_{\alpha} t_{a}^{\alpha-1} t$ for $t_{a} \gg t$ to the age-independent asymptotic form $\left\langle x^{2}(t)\right\rangle \simeq K_{\alpha} t^{\alpha}$ for $t_{a} \ll t[14,19]$. Thus, when $t_{a}$ is of the same order as $t$, clear aging effects appear. At the same time, $t_{a}$ shows up merely as an algebraic prefactor in the corresponding time averages [19].

On the semiaxis with $\delta$-initial condition at $x=0$, the FPTD to the point $x_{0}$ of this CTRW process reads

$$
\wp(t)=\left(\frac{K_{\alpha}}{x_{0}^{2}}\right)^{1 / \alpha} l_{\alpha / 2}\left(\left[\frac{K_{\alpha}}{x_{0}^{2}}\right]^{1 / \alpha} t\right) \simeq \frac{x_{0} / K_{\alpha}^{1 / 2}}{t^{1+\alpha / 2}}
$$

in terms of the one-sided Lévy stable law $l_{\alpha / 2}(t)$ [20]. Its asymptotic expansion shows the power-law decay $t^{-1-\alpha / 2}$ characteristic for unbiased subdiffusion [3]. Equation (1) follows from the Laplace image [21] $\wp(u)=\exp \left(-x_{0} u^{\alpha / 2} K_{\alpha}^{1 / 2}\right)$ derived in Ref. [22]. In the Brownian limit $\alpha=1$, the FPTD reduces to the familiar Lévy-Smirnov law with asymptote $\wp(t) \simeq x_{0} /\left[K_{1} t^{3 / 2}\right]$ exhibiting the famed Sparre-Andersen $3 / 2$ universality [23]. 
To extend this result for an aged system $\left(t_{a}>0\right)$, we use the propagator described by the aging CTRW [14,19],

$$
P_{a}(k, s, u)=P_{0}(s, u)+h(s, u)\left[u+K_{\alpha} u^{1-\alpha} k^{2}\right]^{-1},
$$

in Fourier-double Laplace representation $P_{a}\left(x, t_{a}, t\right) \rightarrow$ $P(k, s, u)$ [24]. Its second term contains the Fourier-Laplace image $P(k, u)=1 /\left(u+K_{\alpha} u^{1-\alpha} k^{2}\right)$ of the nonaged CTRW [3]. The additional component in Eq. (2) is the density $h\left(t_{a}, t\right)$ for the so-called forward waiting (or recurrence) time $t$ for the occurrence of the first step in the random walk process, after the system aged for $t_{a}$ originally derived by Godrèche and Luck [13]. Due to the lack of a characteristic scale of $\psi$, increasingly longer trapping times occur while the system evolves. Typically, after the aging period, the system is arrested in such a long trapping state. This changes the statistics of the first step to occur, as quantified by the distribution $h$. The double Laplace transform of $h$ is $h(s, u)=$ $[\psi(s)-\psi(u)] /\{(u-s)[1-\psi(s)]\}[13,14,19]$, from which the asymptotic behavior $h\left(t_{a}, t\right) \simeq 1 /\left(t_{a}^{1-\alpha} t^{\alpha}\right)$ in the aging regime $t_{a} \gg t$ shows that in the aged system the statistics for the forward waiting time $t$ is much longer tailed than in $\psi(t)$ for the nonaged process. Finally, in Eq. (2), $P_{0}(s, u)=$ $[1-\operatorname{sh}(s, u)] /(s u)$ is the Laplace transform of the probability $P_{0}\left(t_{a}, t\right)$ that no step occurs up to time $t$. The splitting into a discrete part for completely immobile particles and a continuous portion weighted by the density $h$ is typical for aging CTRW processes [19].

We consider the scenario that the walker moves in space and, after an aging period $t_{a}$, an absorbing site is introduced, placed at a distance $x_{0}$ from the walker independently of its initial position. This is the relevant scenario when the travel distance per time of aged fluorescent particles is measured or for charge carriers when the electrical field is switched on after the aging period. The case of initially fixed $x_{0}$ is studied elsewhere [25].

Using the standard method of images [23,26] and the subordination trick $[3,27]$, we find the general closed form

$$
\wp_{a}\left(t_{a}, t\right)=\left(\frac{K_{\alpha}}{x_{0}^{2}}\right)^{1 / \alpha} h\left(t_{a}, t\right) \otimes l_{\alpha / 2}\left[\left(\frac{K_{\alpha}}{x_{0}^{2}}\right)^{1 / \alpha} t\right]
$$

for the FPTD, where $\otimes$ denotes a Laplace convolution. This form is very useful for numerical evaluation. The exact expression for $\wp_{a}\left(t_{a}, t\right)$ involves a double-infinite power series [25]. In the long-time limit $(t \rightarrow \infty)$, we find the three scaling regimes,

$$
\wp_{a}\left(t_{a}, t\right) \simeq \begin{cases}t_{a}^{\alpha-1} t^{-\alpha}, & t_{a} \gg t \\ t_{a}^{\alpha} t^{-1-\alpha}, & t_{a} \ll t \ll t^{\star} \\ x_{0} K_{\alpha}^{-1 / 2} t^{-1-\alpha / 2}, & t^{\star} \ll t,\end{cases}
$$

depending on the severity of the aging, where the time scale $t^{\star}=t_{a}^{2}\left\{\sqrt{K_{\alpha}} \Gamma(-\alpha / 2) /\left[x_{0} \Gamma(\alpha) \Gamma(1+\alpha)\right]\right\}$ contains the initial condition $x_{0}$ and $K_{\alpha}$. Thus, when aging is weak, the asymptotic behavior of the nonaged process from Eq. (1) is preserved. Remarkably, once the aging becomes more pronounced, the competition between the magnitudes of the measurement time $t$ and the aging time $t_{a}$ effects a change of the scaling exponent of $t$ from $1+\alpha / 2$ to $1+\alpha$ at intermediate
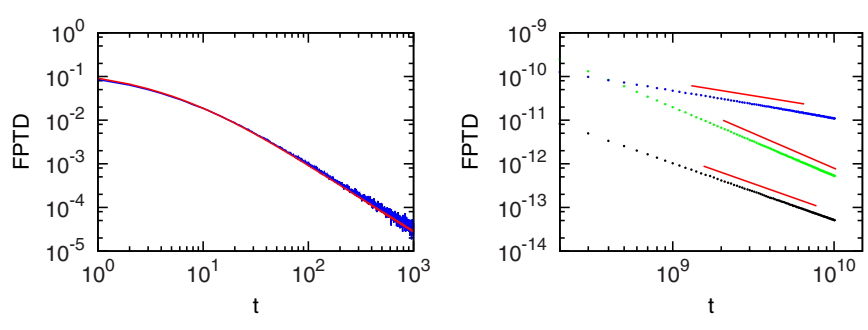

FIG. 1. (Color online) Semi-infinite domain. Left: CTRW simulation (blue) and analytical result (red) for $\alpha=0.75, t_{a}=100$, $x_{0}=1$, and $K_{\alpha}=0.5$. Right: Different scaling regimes in the long-time limit for $t_{a}=0.1$ (black, bottom), $t_{a}=10^{7}$ (green, middle), and $t_{a}=10^{11}$ (blue, top), with $\alpha=0.6, x_{0}=1, K_{\alpha}=0.2$.

values of $t_{a}$, and a further change to $\alpha$ under strong aging conditions. The crossover between these scaling regimes is a key signature of scale-free CTRW processes. Figure 1 on the left demonstrates excellent agreement of our analytical result (3) with simulations of the CTRW process with trapping time density $\psi(\tau)$ [28]. On the right of Fig. 1, we confirm the existence of the three different scaling regimes of $\wp(t)$ predicted by Eq. (4), again observing excellent agreement. Note, however, that in order to see all three regimes, the variation of $t_{a}$ needs to be quite large. Thus, depending on the physical system and the experimental technique, all three regimes may not be detectable. As the first crossover in Eq. (4) increases the magnitude of the scaling exponent while the second crossover decreases it again, the FPTD behavior nevertheless provides an alternative method to deduce the age $t_{a}$ of an aging system. The discovery of three distinct scaling regimes and the dependence of the FPTD on the aging time $t_{a}$ are our first main results.

Note that the results obtained here for CTRWs are fundamentally different from the first passage in long-range correlated Gaussian processes [29] with only transient aging [30]. It will be interesting to see whether similar crossovers also occur in other weakly nonergodic systems, such as diffusion processes with space-dependent diffusion coefficient [31] or scaled Brownian motion [32].

Finite domain. For the first passage to the boundaries of a finite domain, the asymptotic scaling of the nonaged FPTD is $\wp(t) \simeq x_{0}^{2} K_{\alpha}^{-1} t^{-1-\alpha}$ [22], i.e., the decay is steeper than in the semi-infinite case. Due to the divergence of the trapping time scale $\langle\tau\rangle$, however, the mean first-passage time still diverges, in contrast to the Brownian case $(\alpha=1)$, for which $\wp(t)$ has an exponential cutoff [23]. For the aged system, we again derive $\wp_{a}\left(t_{a}, t\right)$ via the images method. From a convolution similar to Eq. (3), we find an exact solution in terms of a power series with Lerch functions [25]. The long-time scaling

$$
\wp_{a}\left(t_{a}, t\right) \simeq \begin{cases}t_{a}^{\alpha-1} t^{-\alpha}, & t_{a} \gg t \\ {\left[x_{0}^{2} /\left(2 K_{\alpha}\right)+t_{a}^{\alpha} / \Gamma(1+\alpha)\right] t^{-1-\alpha},} & t_{a} \ll t\end{cases}
$$

emerges, where this time we only observe two scaling regimes in the measurement time $t$ : at weak aging, the scaling exponent is $1+\alpha$, which changes to $\alpha$ at strong aging. Concurrently, the aging time does not appear explicitly as long as $t_{a} \ll$ $\left[x_{0}^{2} \Gamma(1+\alpha) /\left(2 K_{\alpha}\right)\right]^{1 / \alpha}$. At intermediate aging, the prefactor 

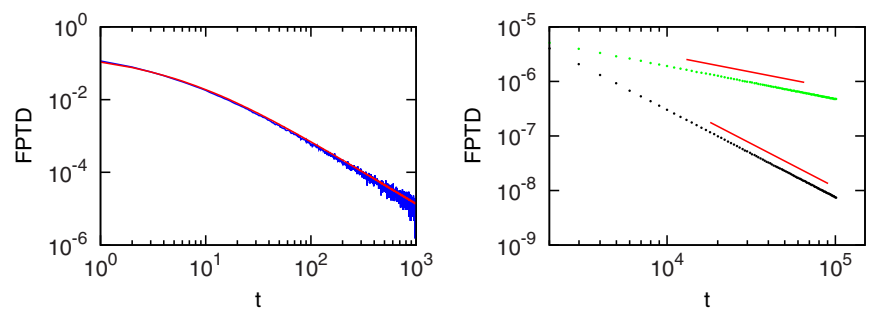

FIG. 2. (Color online) Finite domain. Left: CTRW simulation (blue) and analytical result (red) for $\alpha=0.75, t_{a}=100, x_{0}=1$, and $K_{\alpha}=0.5$. Right: Different scaling regimes in the long-time limit for $t_{a}=0.1$ (black, bottom) and $t_{a}=10^{7}$ (green, top), with $\alpha=0.6$, $x_{0}=1, K_{\alpha}=0.2$.

$t_{a}^{\alpha}$ enters, while for strong aging, it changes to $t_{a}^{\alpha-1}$. Figure 2 shows excellent agreement with these results. The behavior of the aged FPTD on a finite domain is our second important result.

Biased diffusion. In many physical systems, the motion of the particle is biased by an external force, for instance, the electrical field acting on the charge carriers in the amorphous semiconductor of Ref. [1] or a subsurface water stream dragging along the dissolved tracer chemicals in groundwater aquifers [4]. To explore the effects of an external bias on the FPTD, we now add the force $F$ to the dynamics. In the classical Brownian case, a bias towards the absorbing boundary leads to an exponential decay of the associated FPTD, with the mean first-passage time $x_{0} m \eta / F$, where $\eta$ is the friction coefficient. In the nonaged case with a scale-free distribution $\psi(\tau)$ of trapping times, the FPTD has the power-law form $\wp(t) \simeq x_{0} t^{-1-\alpha}$ and the mean first-passage time diverges. When the system is aged, the method of images with an appropriate correction factor still applies [23] and we find the FPTD,

$$
\wp_{a}\left(t_{a}, t\right)=h\left(t_{a}, t\right) \otimes\left\{\otimes_{k=1}^{\infty} g_{k}(t)\right\},
$$

as a multiple convolution $\otimes_{k=1}^{n} g_{k}(t)=g_{1} \otimes g_{2} \otimes \cdots \otimes g_{n}(t)$ of the function $g_{k}(t)=\mathcal{D}(\alpha, k) l_{k \alpha}[\mathcal{D}(\alpha, k) t][33]$ with

$$
\mathcal{D}(\alpha, k)=\left[\left(\frac{2 T}{F}\right)^{2 k-1}\left(\frac{2}{K_{\alpha}}\right)^{k} \frac{\Gamma(3 / 2)}{\Gamma(3 / 2-k) k !}\right]^{-1 / k \alpha} .
$$

Here, the temperature $T$ enters due to the competition between the force $F$ and the thermal energy $k_{B} T$ included through the generalized Einstein-Stokes relation $K_{\alpha}=k_{B} T /\left(m \eta_{\alpha}\right)$, where $\eta_{\alpha}$ is the generalized friction coefficient [34]. The exact solution involves a double series with generalized regularized hypergeometric functions [25], from which two scaling forms can be distinguished in the limit of long measurement times,

$\wp_{a}\left(t_{a}, t\right) \sim \begin{cases}t_{a}^{\alpha-1} t^{-\alpha}, & t_{a} \gg t \\ {\left[x_{0} T /\left(F K_{\alpha}\right)+t_{a}^{\alpha} / \Gamma(1+\alpha)\right] t^{-1-\alpha},} & t_{a} \ll t .\end{cases}$

Similar to Eq. (5) for a finite domain, we obtain the crossover from the $t$ scaling with exponent $1+\alpha$ to $\alpha$ with increased aging, while the aging time appears explicitly for intermediate and strong aging. Figure 3 shows excellent agreement of our exact result with simulations.
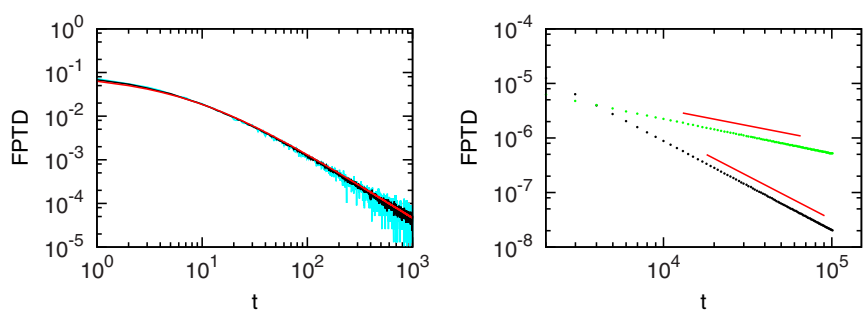

FIG. 3. (Color online) Semi-infinite domain, biased case. Left: Simulations of quenched trap model (cyan) and CTRW (black), and analytical result (red), for $\alpha=0.5, t_{a}=100, F=1, T=0.5, x_{0}=$ $0.2, \tau_{0}=10^{-4}$. Right: Different scaling regimes for $t_{1}=0.1$ (black, bottom) and $t_{a}=10^{7}$ (green, top), for $\alpha=0.6, x_{0}=1$, and $K_{\alpha}=0.2$. In the quenched trap model, we averaged over $10^{5}$ realizations.

Quenched trap model. The quenched trap model [16] is often used as a physical model for CTRW processes with scale-free trapping times. In this lattice model, each site is assigned a random energy value, taken from an exponential density $p(E)=T_{g}^{-1} \exp \left(-E / T_{g}\right)$, where we set the Boltzmann constant to unity. $T_{g}$ is the system-specific "glass" temperature setting the scale for the energy distribution $p(E)$ [16]. A sample realization for such a quenched energy landscape is displayed in Fig. 4. At lattice site $x$, the walker faces the trap energy $E_{x}$ and needs to escape by thermal fluctuations (Kramers escape). After escaping a trap, the walker jumps to one of the two nearest sites and is trapped again; see the schematic in Fig. 4. According to the Arrhenius law, the trapping time at $x$ becomes $\tau_{x}=\tau_{0} \exp \left(E_{x} / T\right)$, where $T$ is the bath temperature and $\tau_{0}$ is an inverse microscopic rate of escape attempts. The combination of the density $p(E)$ with the Arrhenius law yields the long-tailed distribution of trapping times, $\psi(\tau)=\mu \tau_{0}^{\mu} \tau^{-1-\mu}$, with the scaling exponent $\mu=T / T_{g}$. When $T<T_{g}$, the quenched trap model thus leads to a power-law trapping time density with diverging characteristic trapping time $\langle\tau\rangle$ [16].

In the CTRW model, individual trapping times $\tau$ all have the same distribution $\psi$ but are independent variables, such that the system is renewed each time a new trapping time is drawn from $\psi$. This annealed form of the disorder contrasts the quenched nature of the trap model. A walker that leaves a trap may revisit it during later jumps, and the ensuing random walk thus exhibits correlations and is not a renewal process. Such correlations are irrelevant in dimensions three and higher,
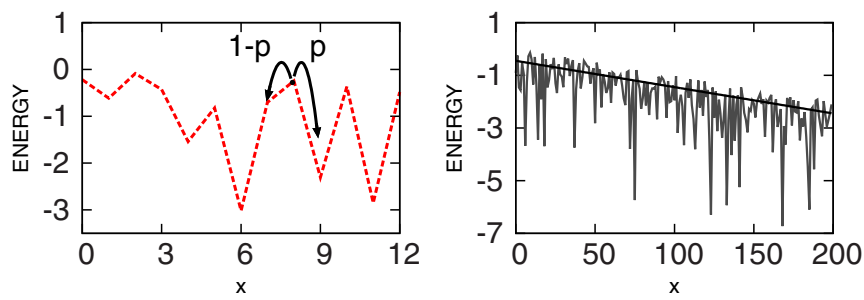

FIG. 4. (Color online) Left: Schematic motion in quenched trap model with energy-dependent transition probabilities $1-p$ and $p$ for moving to the left and right. Right: Realization of the tilted quenched trapped model with $T_{g}=1, F=1$, and $a=10^{-3}$. The lattice constant here is 2 , while we chose 0.01 in Fig. 3. 
as random walks are transient. To avoid such correlations in lower dimensions, we equip the quenched landscape with the external bias force $F$ [35]. This force tilts the quenched trap landscape (Fig. 4) and thus minimizes the likelihood that the walker returns to previously visited sites [16].

The result for the FPTD obtained from the simulations of the quenched trap model matches excellently with both the exact result and the simulation of the annealed CTRW model, as shown in Fig. 3. We thus expect that the first-passage dynamics of an aged particle to a surface in unbiased quenched trap landscapes in three dimensions can be described by results (4) or (5), depending on whether the volume is finite or semiinfinite.

Conclusion. In this Rapid Communication, we studied the first-passage dynamics of an aging diffusion process. In the CTRW model with scale-free trapping time distribution, we showed how aging changes the scaling exponent of the measurement time $t$ and how the aging time $t_{a}$ appears in the FPTD. In the semi-infinite case, we revealed three distinct scaling regimes, while on a finite domain and in the biased case, two scaling regimes appear. Our exact results were demonstrated to agree perfectly with simulations of the CTRW process. In the biased case, the first-passage dynamics measured in the quenched trap model showed excellent agreement with the CTRW approach.

A general feature observed in our results is that aging significantly reduces the efficiency of the first passage, as seen by the decrease of the slope of the FPTD in an aged system. This reflects a general property of processes with scale-free trapping times: as the system evolves, longer and longer trapping times appear on average and lead to a slowing down of the dynamics. For free subdiffusion, this corresponds to an effectively time-dependent diffusivity $K_{\text {eff }} \simeq t^{\alpha-1}$. In the quenched trap model, this would correspond to the particle finding an ever deeper trap during its motion across the energy landscape.

The FPTD represents one of the fundamental concepts in stochastic processes. In many systems, it is fairly easy to experimentally or numerically record the first-passage dynamics. Our findings presented here for aged systems complement the classical results for the first-passage dynamics in nonaged systems governed by scale-free trapping times. In condensedmatter systems, such as amorphous semiconductors, aging is a relevant concern for applications, as it significantly changes the first-passage dynamics and thus the signatures of the electrical current. As the age of amorphous semiconductors can be reliably controlled in experiment over several decades [18], such systems would be ideal to further test the CTRW model for charge carrier transport. In groundwater systems, observation windows span many decades [36] and, due to scale-free trapping time distributions [4], aging would cause a decreased release of chemicals. In biological cells, aging effects lead to the progressive immobilization of particles with potentially relevant biological function in diffusion-limited scenarios $[5,11,19]$. In these biological systems, more than four time decades in the dynamics can be resolved [6] such that sufficient information is available to determine their age. Finally, also for quantum dots, the blinking dynamics can be resolved sufficiently to observe aging effects in the FPTD [8].

The lack of a characteristic trapping time of both the annealed aging CTRW process or the motion in the quenched energy landscape naturally makes the process nonstationary, a property that in turn is closely related to the nonergodicity of the system [37]. Using relatively simple experimental methods to probe the first-passage statistics of a system, such as the charge carrier transport in amorphous semiconductors, as a function of measurement and aging times would represent a direct way to determine the nonstationarity of a system.

With the rapid advance of single molecule tracking techniques, it has become possible to diagnose experimentally recorded time series from individual particle trajectories with respect to the very stochastic mechanism behind the measured anomalous diffusion by various complementary tools [38-43]. This information of the nature of some particle's dynamics, in particular, whether it is an ergodic or nonergodic motion, then allows one to deduce important consequences for the systems, such as the (ir)reproducibility of experiments or the dynamics of followup processes, for instance, the diffusion limitation of reactions. With the characteristic crossovers between different scaling regimes of the measurement time $t$ and the explicit aging time dependence, the current results provide a powerful complementary tool to probe the underlying stochastic mechanism. Moreover, given a sufficiently wide measurement window, our results allow one to read out the actual age $t_{a}$ of the system from measured first-passage data. The possibility of extracting such information should be extremely useful in the analysis of systems with unknown age.

Acknowledgments. A.G. acknowledges funding through an Alexander von Humboldt Fellowship. R.M. acknowledges funding from the Academy of Finland (FiDiPro scheme).
[1] H. Scher and E. W. Montroll, Phys. Rev. B 12, 2455 (1975).

[2] R. Steyrleuthner et al., Adv. Mater. 22, 2799 (2010).

[3] J.-P. Bouchaud and A. Georges, Phys. Rep. 195, 127 (1990); R. Metzler and J. Klafter, ibid. 339, 1 (2000); J. Phys. A 37, R161 (2004).

[4] B. Berkowitz, A. Cortis, M. Dentz, and H. Scher, Rev. Geophys. 44, RG2003 (2006); H. Scher, G. Margolin, R. Metzler, J. Klafter, and B. Berkowitz, Geophys. Res. Lett. 29, 1061 (2002).

[5] S. M. A. Tabei et al., Proc. Natl. Acad. Sci. USA 110, 4911 (2013); A. V. Weigel, B. Simon, M. M. Tamkun, and D. Krapf, ibid. 108, 6438 (2011).

[6] J.-H. Jeon et al., Phys. Rev. Lett. 106, 048103 (2011).
[7] I. Y. Wong et al., Phys. Rev. Lett. 92, 178101 (2004); Q. Xu, L. Feng, R. Sha, N. C. Seeman, and P. M. Chaikin, ibid. 106, 228102 (2011).

[8] X. Brokmann et al., Phys. Rev. Lett. 90, 120601 (2003); G. Margolin and E. Barkai, J. Chem. Phys. 121, 1566 (2004); F. D. Stephani, J. P. Hoogenboom, and E. Barkai, Phys. Today 62, 34 (2009).

[9] F. Bardou, J.-P. Bouchaud, A. Aspect, and C. Cohen-Tannoudji, Lévy Statistics and Laser Cooling (Cambridge University Press, Cambridge, UK, 2002).

[10] J.-P. Bouchaud, J. Phys. I (France) 2, 1705 (1992); G. Bel and E. Barkai, Phys. Rev. Lett. 94, 240602 (2005); A. Rebenshtok 
and E. Barkai, ibid. 99, 210601 (2007); M. A. Lomholt, I. M. Zaid, and R. Metzler, ibid. 98, 200603 (2007); M. Khoury, A. M. Lacasta, J. M. Sancho, and K. Lindenberg, ibid. 106, 090602 (2011).

[11] E. Barkai, Y. Garini, and R. Metzler, Phys. Today 65, 29 (2012).

[12] C. Monthus and J.-P. Bouchaud, J. Phys. A 29, 3847 (1996); B. Rinn, P. Maass, and J.-P. Bouchaud, Phys. Rev. Lett. 84, 5403 (2000).

[13] C. Godrèche and J. M. Luck, J. Stat. Phys. 104, 489 (2001). Compare also N. H. Bingham, Adv. Appl. Prob. 7, 705 (1975); N. H. Bingham, C. M. Goldie, and J. L. Teugels, Regular Variation (Cambridge University Press, Cambridge, UK, 1987).

[14] E. Barkai and Y.-C. Cheng, J. Chem. Phys. 118, 6167 (2003).

[15] Certain aging effects persist for $1<\alpha<2$ when $\langle\tau\rangle$ is finite but $\psi$ has diverging fluctuations: P. Allegrini et al., Phys. Rev. E 66, 015101 (2002); P. Allegrini, G. Aquino, P. Grigolini, L. Palatella, and A. Rosa, ibid. 68, 056123 (2003).

[16] E. M. Bertin and J.-P. Bouchaud, Phys. Rev. E 67, 026128 (2003); S. Burov and E. Barkai, Phys. Rev. Lett. 98, 250601 (2007).

[17] E. Barkai, Phys. Rev. Lett. 90, 104101 (2003); T. Geisel and S. Thomae, ibid. 52, 1936 (1984); G. Radons, Adv. Solid State Phys. 38, 439 (1999).

[18] M. Schubert et al., Phys. Rev. B 87, 024203 (2013).

[19] J. H. P. Schulz, E. Barkai, and R. Metzler, Phys. Rev. Lett. 110, 020602 (2013); Phys. Rev. X 4, 011028 (2014).

[20] B. D. Hughes, Random Walks and Random Environments (Oxford University Press, Oxford, UK, 1995), Vol. 1.

[21] The Laplace transform of a function $f(t)$ is defined as $f(u)=$ $\mathscr{L}\{f(t) ; t \rightarrow u\}=\int_{0}^{\infty} f(t) \exp (-u t) d t$.

[22] R. Metzler and J. Klafter, Physica A 278, 107 (2000).

[23] S. Redner, A Guide to First-passage Processes (Cambridge University Press, Cambridge, UK, 2001).

[24] This transform is defined in terms of $P_{a}(k, s, u)=$ $\mathscr{L}\left\{\mathscr{L}\left\{\int_{-\infty}^{\infty} P_{a}\left(x, t_{a}, t\right) e^{i k x} d x ; t_{a} \rightarrow s\right\} ; t \rightarrow u\right\}$.

[25] H. Krüsemann, A. Godec, and R. Metzler (unpublished).

[26] This method breaks down for long-range correlated Gaussian processes [29] and Lévy flights. See, e.g., A. Zoia, A. Rosso, and M. Kardar, Phys. Rev. E 76, 021116 (2007); A. V. Chechkin et al., J. Phys. A 36, L537 (2003).

[27] J. Klafter and I. M. Sokolov, First Steps in Random Walks: From Tools to Applications (Cambridge University Press, Cambridge, UK, 2011).
[28] The CTRW simulations were performed in continuous space and time. The jump lengths were chosen from a Gaussian distribution with zero mean and unit standard deviation. This means that roughly $16 \%$ of the random walkers reach the boundary after one step. The scaling parameter $\tau_{0}$ in the waiting time distribution $\psi(\tau) \simeq \tau_{0}^{\alpha} / \tau^{1+\alpha}$ is chosen such that $K_{\alpha}=\sigma^{2} /\left[2 \tau_{0}^{\alpha}\right]=0.5$.

[29] S. N. Majumdar, A. Rosso, and A. Zoia, Phys. Rev. Lett. 104, 020602 (2010); see also J.-H. Jeon, A. V. Checkin, and R. Metzler, Europhys. Lett. 94, 20008 (2011).

[30] J. Kursawe, J. H. P. Schulz, and R. Metzler, Phys. Rev. E 88, 062124 (2013).

[31] A. G. Cherstvy, A. V. Chechkin, and R. Metzler, New J. Phys. 15, 083039 (2013); A. G. Cherstvy and R. Metzler, Phys. Chem. Chem. Phys. 15, 20220 (2013); P. Massignan, C. Manzo, J. A. Torreno-Pina, M. F. García-Parajo, M. Lewenstein, and G. J. Lapeyre, Jr., Phys. Rev. Lett. 112, 150603 (2014).

[32] S. C. Lim and S. V. Muniandy, Phys. Rev. E 66, 021114 (2002); F. Thiel and I. M. Sokolov, ibid. 89, 012115 (2014).

[33] Here $l_{k \alpha}(z)$ denotes a function that is formally defined in terms of its Laplace image as $l_{k \alpha}(u)=\exp \left(-u^{k \alpha}\right)$. For $k \alpha>1$, this is no longer a one-sided stable law.

[34] R. Metzler, E. Barkai, and J. Klafter, Phys. Rev. Lett. 82, 3563 (1999).

[35] S. Burov and E. Barkai, Phys. Rev. E 86, 041137 (2012).

[36] J. W. Kirchner, X. Feng, and C. Neal, Nature (London) 403, 524 (2000).

[37] S. Burov, R. Metzler, and E. Barkai, Proc. Natl. Acad. Sci. USA 107, 13228 (2010).

[38] M. Magdziarz, A. Weron, K. Burnecki, and J. Klafter, Phys. Rev. Lett. 103, 180602 (2009); K. Burnecki et al., Biophys. J. 103, 1839 (2012).

[39] S. Condamin, V. Tejedor, R. Voituriez, O. Bénichou, and J. Klafter, Proc. Natl. Acad. Sci. USA 105, 5675 (2008).

[40] I. M. Sokolov, Soft Matter 8, 9043 (2012); F. Thiel, F. Flegel, and I. M. Sokolov, Phys. Rev. Lett. 111, 010601 (2013).

[41] V. Tejedor et al., Biophys. J. 98, 1364 (2010); J.-H. Jeon, E. Barkai, and R. Metzler, J. Chem. Phys. 139, 121916 (2013); A. Cherstvy, A. V. Chechkin, and R. Metzler, Soft Matter 10, 1591 (2014).

[42] T. Albers and G. Radons, Europhys. Lett. 102, 40006 (2013); M. Heidernaetsch, M. Bauer, and G. Radons, J. Chem. Phys. 139, 184105 (2013).

[43] A. M. Berezhkovskii, L. Dagdug, and S. M. Bezrukov, Biophys. J. 106, L09 (2014); M. Saxton, ibid. 103, 2411 (2012); 72, 1744 (1997). 